\title{
The Use of Point-of-Care Ultrasound for Arthrocentesis Among Emergency Medicine Residents
}

This article was published in the following Dove Press journal: Open Access Emergency Medicine

\author{
Josie Acuna (iD \\ Adrienne Yarnish \\ Elaine Situ-LaCasse \\ Richard Amini \\ Srikar Adhikari
}

Department of Emergency Medicine, University of Arizona, Tucson, AZ, USA
Correspondence: Josie Acuna Department of Emergency Medicine, The University of Arizona Medical Center, PO Box 245057, Tucson, AZ, 85724-5057, USA

Tel +I $760485-1330$

Email jacuna@aemrc.arizona.edu
Introduction: The objective of this study is to determine if EM resident physicians are able to successfully utilize POCUS to perform an arthrocentesis in the ED. This is a retrospective review of ED patients who received an ultrasound-guided or ultrasound-assisted arthrocentesis performed in the ED over a 6-year period by an EM resident physician.

Methods: This was a retrospective review of ED patients who received an ultrasoundguided or ultrasound-assisted arthrocentesis performed in the ED over a 6-year period by an EM resident physician. An ED POCUS database was reviewed for POCUS examinations where an arthrocentesis was performed. Electronic medical records were then reviewed for demographic characteristics, history, physical examination findings, ED course, additional imaging studies, and the impact of the POCUS study on patient care and disposition.

Results: A total of 101 POCUS examinations of patients were included in the final analysis. The POCUS examinations and procedures were performed by 59 different EM residents at various levels of training. Overall, 92.1\% (93/101) of the procedures were successful. When assessing for image quality, 98/101 (97\%) had recognizable structures at minimum. The majority of the patients $(84 / 101,83.2 \%)$ received additional imaging of the affected joint. In the minority of cases $(23 / 101,22.8 \%)$, the ultrasound-assisted approach was utilized, while $78 / 100(77.2 \%)$ utilized the ultrasound-guided approach. For the studies that utilized the ultrasound-guided approach, the quality of needle visualization was determined to be "good" 40/78 (51.3\%).

Conclusion: EM resident physicians are able to utilize POCUS to perform an arthrocentesis in the ED. Further research is encouraged to determine whether having residents utilize POCUS to perform an arthrocentesis has a significant impact on outcomes and patient care. Keywords: ultrasound, ultrasonography, emergency medicine, arthrocentesis, education, point-of-care

\section{Introduction}

Arthrocentesis is an important procedure that is often used to determine the underlying etiology of monoarthropathy of a joint and is commonly performed in the emergency department (ED). By successfully obtaining synovial fluid for analysis, providers are better able to distinguish between less threatening inflammatory conditions requiring routine pain management versus more urgent, infectious processes requiring prompt intervention. Prior to the more ubiquitous use of point-ofcare ultrasound (POCUS), most ED physicians would perform an arthrocentesis using the landmark approach. However, now it is common that physicians utilize 
POCUS to assist in this procedure. Some have advocated that ultrasound be used for the primary approach with any arthrocentesis. ${ }^{1}$ While there are opposing perspectives on whether emergency physicians should routinely use ultrasonography for ED arthrocentesis, this approach is becoming the preferred method for many. Therefore, it is not surprising to find that arthrocentesis techniques utilizing ultrasound (US) are being taught during residency. Musculoskeletal and procedural US education among Emergency Medicine (EM) residents has been discussed in the literature. Prior studies have shown that EM residents are not only teachable, but overall perform well after didactic sessions aimed at both musculoskeletal US exams and ultrasound-guided procedures. ${ }^{2-6}$ A prior study by Berona et al evaluated EM resident-performed US on cadavers for diagnosis of effusions and compared the success of a landmark-guided approach with an ultrasound-guided technique for arthrocentesis of various joints. $^{7}$ This study found that while EM residents were able to successfully identify joint effusions with US, they were unable to detect significant differences in actual procedural success between the two modalities. Further research is needed to define the role of US for arthrocentesis in the ED.

This is a retrospective review of ED patients who received an ultrasound-guided or ultrasound-assisted arthrocentesis performed in the ED over a 6-year period by an EM resident physician. The objective of this study is to evaluate the performance of EM residents when utilizing POCUS to perform an arthrocentesis. We suggest, based on prior literature, that ED resident physicians would likely be able to be trained and successfully utilize POCUS when performing an arthrocentesis. We predict that residents will be able to able to obtain images with recognizable structures, have adequate needle visualization and overall perform the arthrocentesis successfully.

\section{Materials and Methods}

\section{Study Design and Setting}

This was a retrospective review of patients who received an ED arthrocentesis performed by an EM resident utilizing POCUS over a 6-year period from January 1, 2014 through January 12020 . Approval from the University of Arizona Institutional Review Board was obtained for this study. This study includes ED arthrocentesis performed at two urban academic EDs totaling approximately 110,000 patient visits per year. Both EDs have an ACGME
(Accreditation Council for Graduate Medical Education)accredited Emergency Medicine residency program. One ED has an additional five-year combined Emergency Medicine/Pediatrics residency program and an Emergency Ultrasound fellowship-training program. The residents receive US training per ACGME guidelines. Residents typically enter their first year of residency training with minimal prior US experience. In their first year of training, all residents complete a 2 -week rotation in emergency US where they receive didactics and hands-on experience in basic US technique. Emergency Medicine residents also received annual training sessions on performing an arthrocentesis utilizing POCUS. The training sessions were approximately 1-2 hours long and taught by Emergency Ultrasound fellows or Emergency Ultrasound fellowship-trained faculty. The residents' first session took place in their first year of residency. Students were given an introductory lecture highlighting the technique and benefits of US for arthrocentesis. Residents were walked through a basic protocol for performing the procedure.

Residents were first instructed on how to obtain US images of each of the commonly affected joints; specifically, how to locate the optimal space for identifying and draining the effusion. It was discussed how to optimize the image using controls such as gain and depth. They were shown how to determine the depth of the effusion so that they are able to plan accordingly when choosing needle length for the procedure. Both US-guided and US-assisted techniques were discussed, but residents were encouraged to perform US-assisted procedures when possible. Residents were given a hands-on session practicing needle guidance for instances in which the US-guided technique was used. Needle guidance and enhancement software was not available. Emphasis was placed on enhancing needle visualization with gain and depth settings. The in-plane needle approach was strongly encouraged so as to keep the entire needle in view for the entirety of the procedure. A variety of standard, cart-based US systems were available for use in the ED: Mindray M9 (Shen Zhen, China), Philips CX50 (Amsterdam, The Netherlands), Zonare Ultra (Zonare Medical Systems, Mountain View, California) and Philips Sparq (Philips Healthcare, Andover, Massachusetts).

The decision to perform the arthrocentesis on a patient to further evaluate for potential joint pathology was ultimately at the discretion of the treating EM attending physician. Residents were instructed to save all POCUS examinations 
to a web-based workflow solutions database, Qpath (Q-path, Telexy Healthcare, BC, Canada) so that all studies could undergo quality assurance by either Emergency Ultrasound fellows or Emergency Ultrasound fellowship-trained faculty.

\section{Study Protocol}

The Qpath database was initially queried for eligible subjects who received musculoskeletal POCUS examinations followed by an electronic medical record review. The database complies with relevant data protection and privacy regulations. A chart reviewer performed data abstraction using a standardized data extraction form. The chart reviewer first confirmed whether the POCUS and arthrocentesis was performed by a resident and documented their year in training. The data extraction form also included information about demographic characteristics, history, physical examination findings, ED course, POCUS findings, additional imaging studies, impact of the POCUS examination and arthrocentesis on patient management. Impact on patient management was defined as the ED providers' decision to request consultation, order antibiotics, and the decision to admit or discharge the patient.

The US images in the Qpath database were reviewed by US fellowship-trained emergency physicians to determine image quality, size of effusion and needle visibility. Image quality was based on a scale of $1-5$, with a score of 1 signifying unrecognizable images and a score of 5 signifying optimal imaging (Appendix 1). A quantitative assessment of the size of the effusion was obtained by measuring the largest visualized anteroposterior (AP) diameter of the effusion in the longitudinal plane along the main axis of the joint. The largest AP diameter was used to quantify the extent of the effusion. ${ }^{8}$ Reviewers scored effusions based on the AP diameter using the following: $<5 \mathrm{~mm}$ mild effusion, $5-10 \mathrm{~mm}$ moderate effusion, $>10 \mathrm{~mm}$ severe effusion. Needle visibility was rated as none, adequate (needle partially visualized) or good (full needle visualization throughout the exam).

Descriptive statistics were used to summarize the data. Dichotomous and nominal data were presented as percentage frequency of occurrence.

\section{Results}

There were 108 patients identified in the initial QPathE query. Patients were excluded after the initial chart review if it was determined that the resident did not perform the arthrocentesis or if it was unclear through documentation who performed the procedure. A total of 101 POCUS studies of patients were included in the final analysis. The mean age was 54.24 \pm 21.69 years (range 4-98). There were 75 males and 26 females. Further patient characteristics are described in Table 1.

Eighty-six patients had an edematous joint or a palpable effusion documented on physical exam. The POCUS examinations and procedures were performed by residents at various levels of training (Table 2). Arthrocentesis was performed on various joints, with the majority performed on the knee (Table 3 ).

Images were reviewed for both overall image quality, size of effusion and needle visualization by emergency ultrasound fellowship-trained physicians. When assessing for image quality, none of the studies were determined to have unrecognizable structures and only 3/101 (3\%) studies had "minimally recognizable" structures. The remaining studies reviewed $(98 / 101,97 \%)$ had recognizable

Table I Characteristics of Patients Receiving Arthrocentesis Using Point-of-Care Ultrasound Performed by an Emergency Medicine Resident

\begin{tabular}{|c|c|}
\hline Patient Characteristics & $\begin{array}{l}\text { Number of Patients } \\
\text { (Percentage of Total Patients) }\end{array}$ \\
\hline Male & $75 / 101(74.3)$ \\
\hline Female & $26 / 101$ (25.7) \\
\hline $\begin{array}{l}\text { Pediatric (Less than } 18 \text { years } \\
\text { of age) }\end{array}$ & $4 / 101(4)$ \\
\hline $\begin{array}{l}\text { Geriatric (More than } 65 \\
\text { years of age) }\end{array}$ & $32 / 101$ (31.7) \\
\hline $\begin{array}{l}\text { History of recent trauma to } \\
\text { the affected joint }\end{array}$ & $23 / 101(22.8)$ \\
\hline Immunocompromised & $17 / 10 \mid(16.8)$ \\
\hline $\begin{array}{l}\text { History of Intravenous drug } \\
\text { use }\end{array}$ & $13 / 10 \mid(12.9)$ \\
\hline $\begin{array}{l}\text { Surgical history involving the } \\
\text { affected joint }\end{array}$ & $18 / 101$ (17.8) \\
\hline History of Diabetes Mellitus & $20 / 101(19.8)$ \\
\hline $\begin{array}{l}\text { Pediatric (Less than } 18 \text { years } \\
\text { of age) }\end{array}$ & $4 / 101(4)$ \\
\hline History of Gout & $15 / 101(14.9)$ \\
\hline $\begin{array}{l}\text { History of Osteoarthritis/ } \\
\text { Rheumatoid Arthritis }\end{array}$ & $18 / 10 \mid(17.8)$ \\
\hline $\begin{array}{l}\text { Obesity (Body mass index } \\
\text { greater than } 30 \text { ) }\end{array}$ & $38 / 101(37.6)$ \\
\hline
\end{tabular}


Table 2 Arthrocentesis Outcomes by Level of Resident Training

\begin{tabular}{|l|l|l|}
\hline $\begin{array}{l}\text { Operator } \\
\text { Post- } \\
\text { Graduate } \\
\text { Year }\end{array}$ & $\begin{array}{l}\text { Number of Studies } \\
\text { Performed at Post- } \\
\text { Graduate Year Level }\end{array}$ & $\begin{array}{l}\text { Percent of } \\
\text { Successfully } \\
\text { Performed } \\
\text { Arthrocentesis } \\
\text { Using Point-of-Care } \\
\text { Ultrasound }\end{array}$ \\
\hline 1 & 31 & $87.1 \%$ \\
\hline 2 & 33 & $93.4 \%$ \\
\hline 3 & 35 & $94.3 \%$ \\
\hline 4 & 2 & $100 \%$ \\
\hline
\end{tabular}

structures at minimum. With regard to the size of the effusion, 40/101 (39.6\%) were characterized as "mild", $36 / 101$ (35.6\%) were "moderate", and 25/101 (24.8\%) were "severe". The majority of the patients $(84 / 101$, $83.2 \%$ ) received additional imaging of the affected joint during their encounter. Eighty-four received radiography (XR) and 1 patient had a CT of the affected joint as well. Nine patients had a radiology-performed US of the affected extremity; however, these studies were ordered with an indication to rule out a deep vein thrombosis, not to evaluate a particular joint. The findings on these additional imaging studies were reviewed for the patients in which the arthrocentesis was successful. Of these studies, 25/76 (32.9\%) had additional imaging that did not demonstrate an effusion, yet had an effusion demonstrated on POCUS and went on to have a successful arthrocentesis performed in the ED. All 8 studies where an effusion was found on POCUS examination, but the arthrocentesis was unsuccessful, had an XR performed as well. POCUS

Table 3 Sites at Which Arthrocentesis Was Performed by the Emergency Medicine Resident Utilizing Point-of-Care Ultrasound

\begin{tabular}{|l|l|}
\hline Sites & $\begin{array}{l}\text { Number of Arthrocentesis } \\
\text { Performed }\end{array}$ \\
\hline Knee & 65 \\
\hline Ankle & 18 \\
\hline Elbow & 8 \\
\hline Wrist & 5 \\
\hline Hip & 3 \\
\hline $\begin{array}{l}\text { Ist Metatarsophalangeal } \\
\text { Joint }\end{array}$ & 2 \\
\hline
\end{tabular}

identified only a "mild" effusion in all of these cases. This is compared to radiography, where 6/8 XRs did not have an identifiable effusion per a radiology department read, but rather soft tissue edema.

For the studies that utilized the US-guided approach, the quality of needle visualization was determined. Review of these images found that 40/78 (51.3\%) examinations had "good" needle visualization, 21/78 (26.9\%) had "adequate" visualization and 17/78 (21.8\%) had poor visualization. Of the studies that were determined to have adequate-to-good needle visualization, 60/62 (96.8\%) were successful.

Only six of the effusions were characterized as complex, otherwise the remaining 95 were classified as simple joint effusions. The minority of the examinations $(23 / 101$, $22.8 \%$ ) utilized the US-assisted approach, while 78/100 (77.2\%) utilized the US-guided approach. Overall, 7.9\% $(8 / 101)$ of the procedures were unsuccessful. A procedure was determined to be unsuccessful if less than $1 \mathrm{~mL}$ of aspirate was obtained. Of those that were unsuccessful, 4/8 were attempted using the US-guided technique and four utilized the US-assisted technique. For the studies that utilized the US-guided approach, 68/70 (97.1\%) in-plane were successful. The success rate for out-of-plane was 6/ $8(75 \%)$.

Based off of the findings of the POCUS examination and initial synovial fluid analysis obtained from the arthrocentesis, 30/93 (32.3\%) patients that had successful fluid aspiration were started on antibiotics in the ED. Synovial fluid was sent for culture in 80/93 (86\%) cases where fluid was aspirated successfully and 19 resulted in a positive culture. Synovial fluid was positive for crystals in 20/93 $(21.5 \%)$ cases. While in the ED, 26/101 (25.7\%) patients received orthopedic surgery consults. With regard to final disposition, 28/101 (27.7\%) patients required admission.

\section{Discussion}

Joint pain is one of the most common symptoms among ED patients presenting with musculoskeletal complaints. ${ }^{9}$ The differential diagnosis for joint pain is broad. When a joint effusion is present, an arthrocentesis can be key in obtaining an accurate diagnosis. POCUS has been shown to be a useful adjunct under such circumstances. The use of POCUS has been documented to change management, prompting physicians to perform an arthrocentesis or abandon plans to perform the procedure based on sonographic findings and spare the patient from the procedure's associated risks. ${ }^{2,10}$ Given the demonstrated ability of 
POCUS to aid in arthrocentesis in prior literature, it seems reasonable to assume the potential benefit in training EM residents to utilize POCUS when evaluating for an effusion and performing an arthrocentesis.

As part of their training, residents were first educated on techniques for evaluating various joints suspected of having an effusion. Overall, the residents performed well in terms of visualizing effusions on POCUS examination. The exceeding majority of examinations (97\%) were found to have appropriately visualized the effusion. Furthermore, it seems that the resident-performed US visualized a number of effusions that may have been missed based on exam findings and radiography alone. Physicians often use physical examination findings such as induration, and fluctuance overlying a joint to suggest an effusion, but POCUS can more accurately confirm the presence and location of an effusion, which provides stronger evidence for undergoing the risk, pain, and time of an invasive procedure. ${ }^{11}$ As mentioned previously, $32.9 \%$ of the patients that received additional imaging while in the ED did not demonstrate an effusion, yet had an effusion demonstrated on POCUS and had a successfully performed arthrocentesis. These results highly impacted patient management with regard to the decision to initiate antibiotic treatment, consultation and admissions. Outcomes were similar for those patients who presented without a palpable effusion or edematous joint on exam, findings typically associated with underlying joint pathology. $^{12}$ Of the patients in this study without a documented palpable effusion or edematous joint on physical exam, all were found to have an effusion demonstrated on POCUS examination and had a successfully performed ED arthrocentesis. These findings, in particular, were encouraging that the use of POCUS for arthrocentesis could impact patient care and outcomes.

Traditionally, arthrocentesis is guided by palpation and performed using an anatomical landmark approach. However, prior studies have questioned the accuracy of this method. For example, a systematic review by Wu et al included a total seven studies in their meta-analysis to investigate this claim. The results showed that US-guided knee arthrocentesis were more efficient than the landmark technique alone, whose inaccuracy was found to range from $22 \%$ to $63 \% .^{13,14}$ Our study is in keeping with prior literature findings, demonstrating a high overall rate of successful procedures when ultrasound was utilized for arthrocentesis of the knee, as well as other various joints evaluated in our study. While the success rate was high regardless of PGY level, at glance, there appeared to be slight improvement at each subsequent level. However, a larger sample size would be needed to perform an accurate statistical analysis of success rates at each PGY level. Improvement over the years is expected as experience can be an important contributor to accuracy. ${ }^{13,15}$ Throughout their training, residents become more experienced with POCUS technique and develop their overall procedural competency.

Another key outcome was determining whether residents were successful at needle visualization when performing an arthrocentesis. Multiple studies have found that POCUS can aid in patient safety as a tool for procedural needle guidance. ${ }^{2,16}$ Ultrasound needle guidance was found to reduce the number of attempts and decreased patient-reported pain compared to landmark technique and maximizing the amount of fluid removed during an arthrocentesis. Given this support from the prior literature, adequate needle visualization was a key component of the arthrocentesis didactic session the residents received. Emphasis was placed on the US-guided approach during protocol training with full needle visualization throughout the procedure. Despite efforts to encourage this approach during training there were still several $(23 / 101)$ procedures performed using the US-assisted only method. The proportion of these procedures that were unsuccessful $(4 / 23$, $17.4 \%$ ) was notably higher than those performed using the US-guided technique (4/78, 5.1\%). Residents appeared to be more successful when performing the US-guided technique; however, this conclusion is limited by the small number of US-assisted studies to draw data from.

\section{Limitations}

This study has multiple limitations, with one being its retrospective study design. The small sample size of this study is also a notable limitation. It is likely that there were significantly more patient cases in which the arthrocentesis protocol was utilized, but POCUS images were not saved in the QPath database for reviewers to query. This is a notable limitation as one might consider that unsuccessful cases were more likely the ones that were not saved or documented. Another limitation is that multiple attempts at the procedure were not taken into account. Unfortunately, upon chart review, documentation of multiple attempts for the arthrocentesis was inconsistent. Given that one of the studied benefits of US-guided or USassisted procedures is decreasing the number of failed attempts, this type of information would have been of 
interest for this study. Perhaps a prospective study design could lead to stricter adherence to some of these components of protocol, such as saving images to the Qpath database and more complete documentation. The results could also have been of more value if there were a similar number of attempted arthrocentesis procedures performed in the ED during the same period without the use of POCUS to evaluate for comparable outcomes.

There were also limitations when it came to quantifying joint effusions. Despite the popularity of US in evaluating joint effusions, there is a lack of consensus on guidelines regarding measuring and quantifying joint effusions. Existing literature appears to be predominantly focused on the knee. Our study utilized portions of a technique described in Kakati et al. ${ }^{8}$ However, this was a study that reviewed the role US played in quantifying the size of effusions found only in the knee. We extrapolated portions of this technique to be used in quantifying effusions in other joints as well. Similarly, there is also not a standardized approach for evaluating the quality of needle visualization.

As discussed, the results of any additional imaging were compared to the documented POCUS findings. Unfortunately, none of these patients received magnetic resonance imaging (MRI) of the affected joint, which is widely accepted as the gold-standard imaging modality for identifying and intraarticular disorder. For detecting the presence of an effusion, MRI has been shown to detect as little as 1 milliliter in joints such as the knee and ankle; superior to sonography or radiography. ${ }^{17-19}$ In our study, the vast majority of patients who had additional imaging received radiography as an additional imaging modality. This is typical of many ED settings as MRI is often not widely available for musculoskeletal complaints, particularly those of low acuity.

\section{Conclusions}

Despite limitations, this study suggests that EM residents can be trained and effectively utilize POCUS to perform an arthrocentesis in the ED. Residents performed well in terms of accurately obtaining high-quality images, as well as successfully performing the procedure. Further research is encouraged to determine whether having residents utilize POCUS to perform arthrocentesis has a significant impact on outcomes and patient care.

\section{Funding}

There is no funding to report.

\section{Disclosure}

The authors have no conflicts of interest to report.

\section{References}

1. Gottlieb M, Alerhand S. Ultrasound should be considered for all arthrocentesis. Ann Emerg Med. 2020;75:261-262. doi:10.1016/j. annemergmed.2019.04.018

2. Situ-LaCasse E, Grieger RW, Crabbe S, et al. Utility of point-of-care musculoskeletal ultrasound in the evaluation of emergency department musculoskeletal pathology. World $J$ Emerg Med. 2018;9:262-266. doi:10.5847/wjem.j.1920-8642.2018.04.004

3. Dodge KL, Lynch CA, Moore CL, Biroscak BJ, Evans LV. Use of ultrasound guidance improves central venous catheter insertion success rate among junior residents. $J$ Ultrasound Med. 2012;31:1519-1526. doi:10.7863/jum.2012.31.10.1519

4. Amini R, Camacho LD, Valenzuela J, et al. Cadaver models in residency training for uncommonly encountered ultrasound-guided procedures. J Med Educ Curric Dev. 2019;6:2382120519885638. doi: $10.1177 / 2382120519885638$

5. Boswell B, Farrow R, Rosselli M, et al. Emergency medicine resident-driven point care ultrasound for suspected shoulder dislocation. South Med J. 2019;112:605-609. doi:10.14423/ SMJ.0000000000001046

6. Wilson CL, Keefe D, Ehmann MR. New ultrasound technology is a useful training adjunct for invasive procedures. AEM Educ Train. 2017;1:363-367. doi:10.1002/aet2.10048

7. Berona K, Abdi A, Menchine M, et al. Success of ultrasound-guided versus landmark-guided arthrocentesis of hip, ankle, and wrist in a cadaver model. Am J Emerg Med. 2017;35:240-244. doi:10.1016/ j.ajem.2016.10.056

8. Kakati P, Sodhi KS, Sandhu MS, Singh S, Katariya S, Khandelwal N. Clinical and ultrasound assessment of the knee in children with juvenile rheumatoid arthritis. Indian J Pediatr. 2007;74:831-836. doi:10.1007/s12098-007-0148-1

9. Derlet RW, Kinser D, Ray L, Hamilton B, McKenzie J. Prospective identification and triage of nonemergency patients out of an emergency department: a 5-year study. Ann Emerg Med. 1995;25:215-223. doi:10.1016/S0196-0644(95)70327-6

10. Pourmand A, Shokoohi H, Maracheril R. Diagnostic accuracy 286 of point-of-care ultrasound in detecting upper and lower extremity fractures: an evidence-based approach. Am $J$ Emerg Med. 2018;36:134-136. doi:10.1016/j.ajem.2017.06.052

11. Adhikari S, Blaivas M. Utility of bedside sonography to distinguish soft tissue abnormalities from joint effusions in the emergency department. J Ultrasound Med. 2010;29:519-526. doi:10.7863/ jum.2010.29.4.519

12. Long B, Koyfman A, Gottlieb M. Evaluation and management of septic arthritis and its mimics in the emergency department. West J Emerg Med. 2019;20:331-341. doi:10.5811/westjem.2018.10.40974

13. Wu T, Dong Y, Song HX, Fu Y, Li JH. Ultrasound-guided versus landmark in knee arthrocentesis: a systematic review. Semin Arthritis Rheum. 2016;45:627-632. doi:10.1016/j.semarthrit.2015.10.011

14. Berkoff DJ, Miller LE, Block JE. Clinical utility of ultrasound guidance for intra-articular knee injections: a review. Clin Interv Aging. 2012;7:89-95. doi:10.2147/CIA.S29265

15. Curtiss HM, Finnoff JT, Peck E, Hollman J, Muir J, Smith J. Accuracy of ultrasound-guided and palpation-guided knee injections by an experienced and less experienced injector using a superolateral approach: a cadaveric study. PMR. 2011;3:507-515. doi:10.1016/j. pmrj.2011.02.020

16. Wiler JL, Costantino TG, Filippone L, Satz W. Comparison of ultrasound-guided and standard landmark techniques for knee arthrocentesis. J Emerg Med. 2010;39:76-82. doi:10.1016/j. jemermed.2008.05.012 
17. Tai AW, Alparslan HL, Townsend BA, et al. Accuracy of cross-table lateral knee radiography for evaluation of joint effusions. AJR Am J Roentgenol. 2009;193(4):339-344. doi:10.2214/AJR.09.2562

18. Jacobson JA, Andresen R, Jaovisidha S, et al. Detection of ankle effusions: comparison study in cadavers using radiography, sonography and MR imaging. AJR. 1998;170:1231-1238. doi:10.2214/ ajr.170.5.9574591
19. Karchevsky M, Schweitzer ME. Accuracy of plain films, and the effect of experience, in the assessment of ankle effusions. Skeletal Radiol. 2004;33:719-724. doi:10.1007/s00256-004-0833-3

\section{Publish your work in this journal}

The Open Access Emergency Medicine is an international, peerreviewed, open access journal publishing original research, reports, editorials, reviews and commentaries on all aspects of emergency medicine. The manuscript management system is completely online

Submit your manuscript here: https://www.dovepress.com/open-access-emergency-medicine-journal and includes a very quick and fair peer-review system, which is all easy to use. Visit http://www.dovepress.com/testimonials.php to read real quotes from published authors. 